\title{
Gute Elternschaft. Zum normativen Gehalt der Indikation in der Reproduktionsmedizin
}

\author{
Giovanni Rubeis
}

Eingegangen: 15. Januar 2020 / Angenommen: 27. April 2020 / Online publiziert: 26. Mai 2020 (C) Der/die Autor(en) 2020

Zusammenfassung Die Möglichkeiten der Reproduktionsmedizin erweitern sich ständig. Bei einigen Maßnahmen assistierter Reproduktion ist es oft unklar, ob eine Indikation vorliegt oder ob diese Maßnahmen als wunscherfüllend anzusehen sind. Die Unterscheidung zwischen medizinisch indizierter Maßnahme und wunscherfüllender Behandlung hängt von dem hier verwendeten Konzept der Indikation ab. Daher kommt dem Konzept der Indikation auf dem Gebiet der Reproduktionsmedizin ein besonderer Stellenwert zu. Dabei fällt auf, dass die Abgrenzung zwischen medizinisch indizierter Behandlung und Wunschbehandlung nicht allein klinisch begründet ist, sondern implizit oder explizit normative Urteile enthält. Der sprachlichkommunikative Aspekt spielt hierbei eine zentrale Rolle. Der übliche Sprachgebrauch von ,indiziert“ suggeriert ein Urteil, das allein auf empirisch-klinischen Fakten basiert, und verdeckt die normativen Urteile, die in das Konzept der Indikation einfließen. Ziel des vorliegenden Beitrags ist es, den normativen Gehalt der Indikation in der Reproduktionsmedizin zu analysieren. Grundlage ist die eingehende Betrachtung des Konzepts der Indikation und der besonderen Stellung der Indikation in der Reproduktionsmedizin. Aus dieser Betrachtung ergibt sich, dass der normative Gehalt der Indikation von einem soziokulturellen Skript bezüglich Elternschaft geprägt ist, das sich v. a. im Leistungsrecht niederschlägt. Dieses Skript enthält gesellschaftliche Anschauungen, Normen und Rollenbilder und kann als ,gute Elternschaft" bezeichnet werden. Das Skript der guten Elternschaft wirkt als Regulativ, das durch leistungsrechtliche Vorgaben über den Zugang zu reproduktionsmedizinischen Maßnahmen bestimmt. Es basiert auf traditionellen heteronormativen Eheund Familienkonzepten und sieht Elternschaft für heterosexuelle, verheiratete Paare vor. Somit ergibt sich der Widerspruch, dass Verfahren der Reproduktionsmedizin,

Dr. G. Rubeis $(\bowtie)$

Institut für Geschichte und Ethik der Medizin, Medizinische Fakultät, Ruprecht-Karls-Universität

Heidelberg, Im Neuenheimer Feld 327, 69120 Heidelberg, Deutschland

E-Mail: giovanni.rubeis@ @istmed.uni-heidelberg.de 
die den Raum für die Fortpflanzung jenseits traditionell-heteronormativer Konzepte öffnen, von ebenjenen Konzepten legitimiert und limitiert werden.

Schlüsselwörter Assistierte Reproduktionstechniken (ART) · Gute Elternschaft • Heteronormativität · Indikation · In-vitro-Fertilisation (IVF) ·

Reproduktionsmedizin

\title{
Good parenting. On the normative implications of indication in reproductive medicine
}

\begin{abstract}
Definition of the problem The options of reproductive medicine are expanding. In some cases, it is unclear whether there is a medical indication for applying procedures of assisted reproduction or whether this application is wish-fulfilling. The distinction between medical indication and wish fulfilment depends on the concept of indication. Thus, the concept of indication has a special status in reproductive medicine. The distinction between medical indication and wish-fulfilling treatment is mostly based on implicit or explicit normative judgements, rather than on mere clinical facts. The use of "indicated" suggests a purely empirical-clinical judgment, whereas the normative judgements behind the concept are concealed. The aim of this paper is to analyze the normative implications of indication in reproductive medicine. Therefore, the concept of indication and its special status within reproductive medicine is scrutinized.

Arguments The analysis shows that the normative implications of indication are tied to a sociocultural script of parenthood, which manifests in the regulations of reimbursement. This script encompasses societal views, norms, and role models, and can be described as "good parenting". The script of good parenting acts as a regulatory instance that determines the access to procedures of assisted reproduction. It is based on traditional heteronormative concepts of marriage and family, and reserves reproduction exclusively for heterosexual, married couples.

Conclusion The sociocultural script of good parenting in reproductive medicine implies a contradiction: Assisted reproduction technologies provide the opportunity of reproduction for individuals outside the heteronormative frame, yet they are legitimized and limited by this exact frame.
\end{abstract}

Keywords Assisted reproduction technologies (ART) - Good parenthood · Heteronormativity · Indication · In vitro fertilization (IVF) - Reproductive medicine

Seit einigen Jahren wird die medizinische Indikation vermehrt diskutiert (Dörries und Lipp 2015; Hunstorfer und Wallner 2016; Wiesing 2017). Dennoch sind nach wie vor mit der Indikation verknüpfte Fragen offen. So werden etwa Bedeutung und Tragweite der Indikation im Kontext einzelner medizinischer Fachgebiete nur selten thematisiert (Dörries 2015). Im vorliegenden Beitrag soll die Rolle der Indikation im Bereich der Reproduktionsmedizin untersucht werden. Gerade in der Reproduktionsmedizin ist die Abgrenzung zwischen medizinisch indizierten und nicht-indizierten 
Maßnahmen häufig umstritten. Nach Ansicht mancher Kommentator*innen entzieht sich die Reproduktionsmedizin den klassischen Zielen ärztlichen Handelns, wie etwa der kurativen Therapie, der Prävention, der Gesundheitsfürsorge oder der palliativen Therapie (Buyx und Hucklenbroich 2009). Daher werden assistierte Reproduktionstechniken (ART) oftmals als Maßnahmen zur Vitaloptimierung und Lebensplanung verstanden (Kettner 2006). In diesem Verständnis fungieren medizinische Maßnahmen als Unterstützung individueller Lebensplanung und unterscheiden sich dadurch von dem Ziel der Heilung, die als regulative Idee der kurativen Medizin zu verstehen ist (Kettner 2018). Somit erscheint die Charakterisierung von ART als medizinisch indiziert oder wunscherfüllend oftmals unklar. Mit jeder Innovation im Bereich der ART wird diese Debatte neu geführt. So wird etwa hinsichtlich der Kryokonservierung von Eizellen zwischen einer medizinischen Indikation zur Fertilitätsprotektion, etwa vor einer Chemotherapie, und dem nicht indizierten ,Social Freezing“ zur Familien- und Lebensplanung unterschieden (Alteri et al. 2019; van der Ven et al. 2017). Auch hinsichtlich des 2016 erstmals durchgeführten Mitochondrien-Transfers wird diskutiert, ob hier eine indizierte Behandlung oder eine Wunschbehandlung vorliegt (Griffiths 2016). Aktuell gibt es eine Debatte um die Frage, inwiefern die Uterustransplantation medizinisch indiziert ist (Bozzaro et al. 2019; Wilkinson und Williams 2016). Im Zentrum dieser Debatten steht die Frage nach der Notwendigkeit der jeweiligen Behandlung und damit verbunden nach der Abgrenzung zwischen medizinisch indizierten Maßnahmen und Wunschbehandlungen. An diesen Beispielen zeigt sich die besondere Stellung des Konzepts der Indikation im Kontext der Reproduktionsmedizin. Auf diesem Gebiet stehen umfangreiche Maßnahmen der Wunschbehandlung zur Verfügung. Zugleich gibt es eine Nachfrage nach Behandlungen, die nicht medizinisch indiziert sind. Somit steht die Abgrenzungsfunktion der Indikation in der Reproduktionsmedizin besonders im Fokus. Dabei zeigt sich auch die zentrale Bedeutung normativer Konzepte für die Festlegung dessen, was als Indikation zu gelten hat. Oftmals lässt sich die Abgrenzung zwischen medizinisch indiziert und wunscherfüllend nicht allein klinisch begründen, sondern enthält implizit oder explizit normative Urteile. Sprachlich wird diese Normativität verdeckt, da der Begriff der Indikation eine rein empirisch-klinische Fundierung suggeriert. Diesen normativen Gehalt der Indikation in der Reproduktionsmedizin sichtbar zu machen, ist Ziel des vorliegenden Beitrags. In einem ersten Schritt werden verschiedene Aspekte der Indikation sowie entsprechende Modelle vorgestellt. In einem weiteren Schritt werden Definition und Stellenwert der Indikation innerhalb der medical community einerseits und im Kontext der rechtlichen Regulierungen andererseits analysiert. Dabei werden die jeweils unterschiedlichen normativen Implikationen herausgearbeitet. Hierbei spielen das unklare Krankheitskonzept der Infertilität sowie das soziale Skript guter Elternschaft eine zentrale Rolle. Abschließend werden die Ergebnisse zusammengefasst.

\section{Indikation: Aspekte und Modelle}

Die medizinische Indikation lässt sich grundsätzlich als Antwort auf die Frage verstehen, was in einem konkreten klinischen Einzelfall $\mathrm{zu}$ tun ist (Hunstorfer und 
Wallner 2016). Dabei erfüllt sie eine handlungsleitende und eine legitimatorische Funktion.

Handlungsleitend ist die Indikation als Bindeglied zwischen der biomedizinischen Analyse des vorliegenden Krankheitsbildes und der Durchführung konkreter Maßnahmen (Marckmann 2015). Sie hat eine Lenkungsfunktion, indem sie die Interaktion von Ärzt*in und Patient*in vom Hilfeersuchen bis zur Durchführung von Therapiemaßnahmen strukturiert (Gahl 2005). Nach diesem Verständnis ist die Indikation zum einen ein Urteil darüber, ob eine bestimmte Maßnahme in einem konkreten Einzelfall angemessen ist (Marckmann 2015). Zum anderen ist sie ein Urteil darüber, wie ein legitimes Behandlungsziel im gegebenen Fall erreicht werden kann (Marckmann 2015). Ärzt*innen beurteilen die geeigneten Mittel nach Maßgabe von evidenzbasiertem Fachwissen, individueller Erfahrung und von Aspekten, die sich auf individuelle Patient*innen beziehen (Patientenspezifika) (Dörries 2015). Medizinisch-fachliche Aspekte wie Symptome, Ätiologie, Pathogenese, Prognose (v.a. bezüglich der Dringlichkeit) sind hier von Bedeutung (Gahl 2005). Die Determinanten der Beurteilung der Mittel sind der Zustand des/der Patient*in, der zu erwartende Verlauf, die Heilungschancen sowie die Wirksamkeit, Effektivität und Effizienz von Verfahren und Maßnahmen (Gahl 2005). Entscheidend ist die Einschätzung einer Maßnahme als sinnvoll, nützlich oder hilfreich (Neitzke 2015).

Legitimatorisch ist die Indikation, indem sie eine der drei Grundvoraussetzungen der Heilbehandlung neben dem Informed Consent und der lege artis-Behandlung darstellt (Hunstorfer und Wallner 2016). Erst durch die Indikationsstellung werden die diagnostischen und therapeutischen Maßnahmen in einem konkreten Einzelfall legitimiert (Marckmann 2015). Durch ein evidenzbasiertes Urteil über Mittel und Ziele der Behandlung ist die Indikation legitimatorische Grundlage medizinischen Handelns (Hunstorfer und Wallner 2016). Durch die Indikation wird festgelegt, welche Optionen Patient*innen vorgelegt werden (Marckmann 2015). Der Bereich dessen, was Ärzt*innen legitimerweise anbieten und Patient*innen legitimerweise nachfragen dürfen, wird damit definiert (Neitzke 2015). Sie erfüllt somit eine wichtige Filterfunktion. Dadurch stellt die Indikation die Grenze der Ansprüche von Patient*innen dar und begrenzt zugleich den Inhalt des ärztlichen Heilauftrags (Marckmann 2015). Nicht-indizierte Maßnahmen sollen nicht, kontraindizierte Maßnahmen dürfen nicht durchgeführt werden (Wiesing 2017). Zu dieser klinischen Legitimation kommt eine sozialrechtliche hinzu. Die Indikationsstellung entscheidet über die solidargemeinschaftliche Kostenübernahme einer medizinischen Leistung (Dörries 2015).

Die Indikation ist keine rein deskriptive Analyse, sondern verbindet empirische Urteile hinsichtlich der geeigneten Mittel mit normativen Urteilen (Marckmann 2015). Letztere beziehen sich zunächst auf Wertvorstellungen innerhalb der $m e$ dical community (Hunstorfer und Wallner 2016). Hier sind das Handlungsziel der Medizin im Sinne des ärztlichen Heilauftrags sowie das damit verbundene Selbstverständnis der Medizin zu nennen. In dieser Hinsicht werden Ziele wie die Heilung von Krankheiten, die Linderung von Leid und die Wiederherstellung bzw. Erhaltung von Gesundheit als legitime Ziele angesehen, während Optimierung und Leistungssteigerung (Enhancement) allgemein nicht als Ziele der Medizin gelten. Somit kommt zur Zweckrationalität der Bewertung geeigneter Mittel das Element 
der Wertrationalität hinzu (Neitzke 2015). Darüber hinaus ist hier der rechtliche, v. a. leistungsrechtliche Rahmen miteinzubeziehen. Auch hier finden sich normative Urteile darüber, welche Personen unter welchen Bedingungen Zugang zu ART und zur solidargemeinschaftlichen Kostenübernahme haben.

Des Weiteren beziehen sich normative Urteile auf den Patientenwillen. Strittig ist jedoch, inwieweit der Patientenwille einen normativen Faktor der Indikation darstellt (Wiesing 2017). Manche Autor*innen gehen davon aus, dass Indikation und Patientenwille sich nicht voneinander trennen lassen (Gahl 2005; Hunstorfer und Wallner 2016). Die Indikation gehe dem Patientenwillen nicht voraus, sondern fließe bereits bei der Festlegung des Therapieziels mit ein (Hunstorfer und Wallner 2016). Demgegenüber steht die Ansicht, wonach die Indikation den Rahmen des medizinisch Möglichen aufzeigt, d.h. die Mittel, um ein von Patient*innen präferiertes Therapieziel zu erreichen (Wiesing 2017). Durch die Indikationsstellung werden die Handlungsoptionen von Ärzt*innen und Patient*innen vorselektiert (Wiesing 2017). Dadurch stellt die Indikation einen Filter für diejenigen Maßnahmen dar, über deren Durchführung entschieden werden soll (Marckmann 2015). Da die Indikation selbst noch keine Entscheidung für die Durchführung einer Maßnahme ist, muss sie von der gemeinsamen Entscheidungsfindung getrennt werden. Erst bei der Entscheidungsfindung kommt der Patientenwille ins Spiel (Wiesing 2017).

Es gibt verschiedene Ansätze, um eine Lösung dieser Streitfrage zu erreichen (Alt-Epping und Nauck 2012; Marckmann 2015; Neitzke 2015; Wiesing 2017). Dabei fällt auf, dass Konflikte besonders zwischen den Werturteilen von Ärzt*innen und den Präferenzen von Patient*innen verortet werden. Es ist jedoch vielmehr davon auszugehen, dass der eigentliche Konflikt nicht primär mit den individuellen Werthaltungen von Ärzt*innen oder den Normen innerhalb der medical community verbunden ist. Vielmehr basieren mögliche Konflikte auf der Diskrepanz zwischen den Präferenzen von Patient*innen und den Normen, welche den (leistungs-)rechtlichen Regelungen zugrunde liegen. Behandelnde sind demnach häufig mit Wünschen von Patient*innen konfrontiert, die biomedizinisch erfüllbar wären und aus Sicht der medical community weitestgehend als legitim gelten können, deren Erfüllung aber nur in wenigen Fällen als im rechtlichen Sinn indiziert gilt. Das bedeutet, dass nicht allein klinische Faktoren auf die Indikationsstellung einwirken, sondern auch normative Faktoren von zentraler Bedeutung sind, und das in höherem Maß als in anderen Bereichen. Allein die Bezeichnung Kinderwunschbehandlung, die in der Reproduktionsmedizin gebräuchlich ist, zeigt an, dass man es hier mit einer spezifischen Situation zu tun hat. Niemand würde von Antitumor-Wunschbehandlung oder einer Transfusions-Wunschbehandlung sprechen. Wie gesehen, gehört es zur legitimatorischen Funktion der Indikation, medizinisch notwendige Maßnahmen von wunscherfüllenden Maßnahmen abzugrenzen. Somit ist zu fragen, inwieweit eine Kinderwunschbehandlung indiziert sein kann und welche normativen Faktoren in der medical community sowie im Recht hierfür eine Rolle spielen. Zu analysieren ist dafür das regulative Rahmenwerk, das die klinische Praxis strukturiert. Dieses Rahmenwerk besteht aus Richtlinien und rechtlichen Bestimmungen, die zum Teil von den Akteuren der medical community gestaltet werden, z. B. in Form von Berufsordnungen. Darüber hinaus wird dieser Rahmen auch von gesellschaftlichen Normen sowie Rechtsregeln gebildet. Die Medizin ist kein von gesellschaftlichen 
Wertvorstellungen isolierter Bereich. Da soziale Normen auch über das rechtliche Rahmenwerk in die Medizin einfließen, lässt sich dieses als durchlässige Membran zwischen dem klinischen Bereich und der Gesellschaft verstehen. Um die normativen Urteile in der Reproduktionsmedizin zu rekonstruieren, werden daher klinische Konzepte sowie Regularien der klinischen Praxis in die Analyse miteinbezogen.

\section{Normative Faktoren der Indikation in der Reproduktionsmedizin}

Die klinischen und leistungsrechtlichen Rahmenbedingungen der Indikationsstellung in der Reproduktionsmedizin verweisen auf die normativen Faktoren, von denen eine Behandlung abhängt. Hier zeigt sich eine Anerkennung des Kinderwunsches bei gleichzeitiger Limitierung.

In der medical community wird der Kinderwunsch als prinzipiell legitim angesehen und der Infertilität wird ein Krankheitswert zugeschrieben, was die Notwendigkeit einer Behandlung impliziert. Die leistungsrechtliche Sonderstellung der Infertilitätsbehandlung und die einschlägigen Debatten zeigen allerdings, dass der Krankheitswert der Infertilität umstritten ist. Daher ist die zugrundeliegende Krankheitstheorie in der Reproduktionsmedizin zu analysieren.

Die leistungsrechtlichen Vorgaben lassen sich als Indikator gesellschaftlicher Einflussfaktoren verstehen. An ihnen lässt sich ablesen, welche Formen der Reproduktion als zulässig erachtet werden und welche Personen als berechtigt gelten. Hier spielen gesellschaftliche Zuschreibungen und Normen, mithin ein soziokulturelles Skript, eine zentrale Rolle. Dabei ist auch die Befähigung zur Elternschaft nach gelungener Reproduktion bereits im Blick. Somit ist das Konzept von Elternschaft zu analysieren, das hinter diesen Werturteilen steht. Die maßgeblichen Faktoren für die Anerkennung und Legitimierung des Kinderwunsches sind demnach das zugrundeliegende Krankheitskonzept für die medical community und das soziokulturelle Skript von Elternschaft für die rechtliche; v. a. leistungsrechtliche Regulierung.

\section{Indikationsstellung in der Reproduktionsmedizin}

Für die Indikationsstellung ist zunächst die Diagnose der Infertilität zentral. Infertilität liegt vor, wenn nach zwölf Monaten ungeschützten Geschlechtsverkehrs keine Schwangerschaft eingetreten ist (BÄK 2018). Die Verwendung von menschlichen Keimzellen für die assistierte Reproduktion ist zulässig, sofern sie nach ärztlicher Beurteilung medizinisch indiziert und der gesundheitliche Schutz von Mutter und Kind gewährleistet ist (BÄK 2018). Es werden eine primäre, eine sekundäre und eine idiopathische Sterilität unterschieden (Zollner 2015). Von einer primären Sterilität spricht man, wenn bislang noch keine Schwangerschaft erfolgt war. Zu den Ursachen gehören die Ovarialinsuffizienz in Form von funktionellen Störungen oder eines Tumors oder die Beeinträchtigung der Spermienkonzentration und -menge sowie der Motilität und Morphologie von Spermien. Eine sekundäre Sterilität liegt vor, wenn es bereits Schwangerschaften, Aborte oder Extrauteringraviditäten gegeben hat. Bei der idiopathischen Sterilität ist die zugrundeliegende Ursache unbekannt. Zulässige therapeutische Maßnahmen sind die hormonelle Zyklusstimulation durch Gabe 
eines Antiöstrogens oder Injektion von Gonadotropinen. Des Weiteren ist die intrauterine Insemination angezeigt, etwa bei mangelnder Spermienqualität. Die Invitro-Fertilisation (IVF) wird bei Tubenverschluss oder Endometriose durchgeführt, nach einer erfolglosen Inseminationsbehandlung oder bei altersbedingter Infertilität. Die intrazytoplasmatische Spermieninjektion (ICSI) ist bei erfolgloser IVF angezeigt (BÄK 2018; Zollner 2015). Zudem kann eine Kryokonservierung von Samenoder Eizellen als Maßnahme der Fertilitätsprotektion vor der Durchführung einer zytotoxischen Chemo- oder Radiotherapie indiziert sein (Beyer et al. 2013). Zu den indizierten Maßnahmen gehört darüber hinaus die Präimplantationsdiagnostik (PID), die im Rahmen einer IVF bei Vorliegen bekannter genetischer Dispositionen, Erkrankungen oder struktureller Chromosomenveränderungen sowie altersbedingter Risiken angezeigt ist (Montag et al. 2020).

\section{Krankheitskonzept}

Da der Infertilität aus klinischer Sicht ein Krankheitswert zugeschrieben wird, liegt es nahe, Reproduktionsfähigkeit als Funktion eines gesunden Organismus zu verstehen. Der Verlust bzw. Mangel der Reproduktionsfähigkeit stellt demnach eine Funktionseinschränkung dar, die einer medizinischen Behandlung bedarf. Dies entspricht der Position der Bundesärztekammer (BÄK 2018) sowie der WHO und weiterer Fachgesellschaften (Maung 2019). Innerhalb der medical community ist der Krankheitswert der Infertilität jedoch umstritten. Die ungewollte Kinderlosigkeit wird von manchen als nachteiliges persönliches Merkmal angesehen, von anderen als eine Normabweichung und Funktionsstörung ohne notwendigen Krankheitswert und schließlich wieder von anderen als eine Krankheit mit Behandlungsnotwendigkeit und damit verbunden dem legitimen Einsatz öffentlicher Ressourcen (Krones et al. 2006). Oftmals wird die Infertilität auch als ein soziales Konstrukt angesehen, hinter welchem eine pronatalistische Agenda verortet wird (Maung 2019). Andere Interpretationen sehen im Kinderwunsch den Ausdruck eines bestimmten Lifestyles, wodurch bei Nichterfüllung zwar von einem beeinträchtigten Lebensplan, nicht jedoch von einer Krankheit gesprochen werden kann (McTernan 2015). Die Besonderheit der Infertilität liegt darin, dass sie zwar eine Funktionseinschränkung darstellt, jedoch der damit verbundene Leidensdruck nicht in jedem Fall gegeben ist (Maung 2019). Manche Personen empfinden es nicht als Nachteil, unfruchtbar zu sein, während andere Infertilität als einen massiven Verlust empfinden (Letherby 2012). Das bedeutet, dass neben der biomedizinischen Komponente der Funktionseinschränkung noch eine psychosoziale Komponente für den Krankheitswert entscheidend ist. Diese Komplexität spiegelt sich in den einschlägigen Definitionen von Infertilität nicht wider, die einen kategorischen Krankheitswert und damit Behandlungsbedürftigkeit suggerieren. Um dieser Komplexität gerecht zu werden, ließe sich Infertilität als biomedizinische Kategorie von ungewollter Kinderlosigkeit als soziologischer Kategorie unterscheiden (Letherby 2012). Damit wäre die psychosoziale Komponente des Krankheitswerts der Infertilität eingefangen, allerdings zu dem Preis, dass auch bislang als nicht notwendig angesehene Maßnahmen wie das Social Freezing indiziert wären. Zu fragen wäre hierbei nach der Gewichtung zwischen biomedizinischer und psychosozialer Komponente. 


\section{Leistungsrechtlicher Rahmen}

Die Indikationsstellung ist maßgeblich für die solidargemeinschaftliche Kostenübernahme der genannten Maßnahmen assistierter Reproduktion. Aus leistungsrechtlicher Sicht können diese Maßnahmen nur im homologen System in Anspruch genommen werden (G-BA 2017). Das bedeutet, dass allein heterosexuelle, verheiratete Paare anspruchsberechtigt sind. Für die assistierte Reproduktion dürfen ausschließlich Ei- bzw. Samenzellen der Ehepartner verwendet werden. Zudem gilt eine Altersbeschränkung für die Kostenübernahme bei Frauen zwischen 25 und 40 Jahren, bei Männern zwischen 25 und 50 Jahren. Des Weiteren gilt eine Einschränkung hinsichtlich der Häufigkeit der Anwendungen. Bezüglich IVF und ICSI sind drei Behandlungszyklen erlaubt, hinsichtlich der intrauterinen Insemination sind es acht Behandlungszyklen. Eine Besonderheit von ART besteht darin, dass diese Maßnahmen leistungsrechtlich nicht als Krankenbehandlung, sondern als Sozialleistung gelten (Hoffmann 2017). Diese Sonderstellung von ART innerhalb der Gesetzlichen Krankenversicherung (GKV) basiert auf einem höchstrichterlichen Urteil und ist dem unklaren Krankheitswert der Infertilität geschuldet. Damit wird zwar der Kinderwunsch als Anspruchsrecht anerkannt, zugleich aber die medizinische Notwendigkeit einer Behandlung abgesprochen.

\section{Soziokulturelles Skript von Elternschaft}

Das soziokulturelle Skript von Elternschaft besteht aus Zuschreibungen, Erwartungen und Normen hinsichtlich der Rolle von Eltern (Faircloth und Gürtin 2018). Dieses Skript enthält zunächst eine legitimatorische Komponente, da Elternschaft als Normalfall angesehen wird, während Kinderlosigkeit einen Mangel oder eine Normabweichung anzeigt (Letherby 2012). Elternschaft gilt allgemein als erstrebenswert und ist für viele Menschen ein Teil ihrer Identitätskonzeption. Des Weiteren sind mit Elternschaft bestimmte Rollenbilder verbunden, die in einem Narrativ kodiert sind, das divergente, manchmal auch widersprüchliche Regeln enthält (Faircloth und Gürtin 2018). Aus den gesetzlichen, v. a. leistungsrechtlichen Regulierungen von ART lässt sich ein heteronormatives Konzept von Elternschaft ablesen. Anspruchsberechtigte Personen für ART sind verheiratete, heterosexuelle Paare. Dies überrascht, da in anderen gesellschaftlichen Kontexten alternative Lebens- und Familienmodelle anerkannt sind. Hinsichtlich von ART ist jedoch das biologische Modell von Elternschaft gegenüber sozialen Modellen dominant. Somit ergibt sich der Widerspruch, dass gerade die Regulierung von ART ein traditionelles Ehe- und Familienkonzept vorschreibt, obwohl ART in vielen Fällen eine Entkoppelung der Fortpflanzung von der biologischen Elternschaft ermöglichen, indem sie etwa auch gleichgeschlechtlichen Paaren Möglichkeiten zur Fortpflanzung bieten (Faircloth und Gürtin 2018). Kritik an diesem Aspekt kommt aus dem akademischen Diskurs (Maxwell et al. 2018) und nicht zuletzt auch aus der medical community selbst. Deutlich wird diese Kritik z.B. in der Stellungnahme der Leopoldina zur Fortpflanzungsmedizin in Deutschland, die unter maßgeblicher Beteiligung von Mediziner*innen verfasst wurde (Leopoldina 2019). Dort findet sich bezüglich des Zugangs zu ART die explizite Aufforderung, Personen nicht aufgrund ihrer sexuellen Orientierung oder des 
Familienstandes zu diskriminieren. Das gilt auch für die Kostenübernahme. Eine Einschränkung der reproduktiven Autonomie als Grundrecht, so die Argumentation, ließe sich nur durch die Verletzung anderer Rechtsgüter rechtfertigen, etwa einen Verstoß gegen die Grundrechte Dritter oder die Menschenwürde. Da die Inanspruchnahme von ART durch unverheiratete oder homosexuelle Paare diese Rechtsgüter nicht verletzt, sei der Eingriff in deren reproduktive Autonomie unzulässig. Auch in der Richtlinie zur Entnahme und Übertragung von menschlichen Keimzellen im Rahmen der assistierten Reproduktion der Bundesärztekammer wird die Anwendung von ART auf homosexuelle Paare nicht gänzlich ausgeschlossen (BÄK 2018). Standesrechtlich ist es demnach zulässig, dass ein lesbisches Paar oder eine alleinstehende Frau eine heterologe Insemination in Anspruch nimmt (Möller und Makoski 2020). Problematisch ist diese Anwendung jedoch, da erstens nicht alle Landesärztekammern diese Vorgabe umsetzen und zweitens die statusrechtlichen Vorgaben zwischen den Bundesländern divergieren. Demnach könnte aus einer heterologen Insemination eine statusrechtliche Verpflichtung entstehen, z. B. eine Unterhaltspflicht (Möller und Makoski 2020). Für schwule Paare besteht keine Möglichkeit der Inanspruchnahme von ART, da die Leihmutterschaft in Deutschland gesetzlich untersagt ist. Das soziale Skript von Elternschaft normiert zudem das Fortpflanzungsalter, was auf klinische Gründe, wie etwa das Risiko für Fehlgeburten ab einem bestimmten Alter zurückgeführt werden kann. Darüber hinaus liegt hier auch eine Wertung hinsichtlich des gesellschaftlich sanktionierten Alters für Elternschaft vor.

In der Limitierung und Legimitierung der Reproduktionsmedizin geht es somit nicht allein um den Fortpflanzungsprozess. Handlungsleitend und legitimierend ist nicht allein das Ziel, Personen mit Kinderwunsch zu behandeln, um Nachkommen zu erzeugen. Vielmehr wirken v. a. im Leistungsrecht normative Urteile darüber, welche Personen legitimerweise Eltern werden dürfen und welche Eigenschaften sie für die Elternrolle besitzen müssen. Somit könnte man von einem Konzept ,guter Elternschaft" sprechen, das normativ voraussetzungsreicher ist als ,bloße Elternschaft“". Gute Elternschaft ist das Regulativ, das über den Zugang zu ART bestimmt. Es basiert auf den Rollenbildern heteronormativer Ehe- und Familienkonzepte. Die Frage, ob der Kinderwunsch behandlungsbedürftig ist, wird somit nicht allein im klinischen Kontext von Krankheitswert und zugrundeliegendem Krankheitskonzept entschieden. Aus der policy-Perspektive ist nicht allein ausschlaggebend, ob Infertilität eine Krankheit ist oder ob ungewollte Kinderlosigkeit den Anspruch auf Behandlung legitimiert, sondern wer diesen Anspruch stellt. Nach diesem Verständnis sind reproduktionsmedizinische Maßnahmen allein bei heterosexuellen, verheirateten Paaren in einem genau definierten Lebensabschnitt indiziert.

\section{Conclusio}

In der Reproduktionsmedizin manifestiert sich die handlungsleitende und legitimatorische Funktion der Indikation in einem Konzept guter Elternschaft, das den Kinderwunsch legitimiert und zugleich limitiert. Dieses Konzept basiert auf einem soziokulturellen Skript, nach welchem gute Elternschaft verheirateten, heterosexuellen Paaren zugeschrieben wird. Dieses letztlich rein biologische Konzept von Eltern- 
schaft steht im Widerspruch zu gesellschaftlich anerkannten Modellen sozialer Elternschaft. Auffällig ist dabei, dass sich die Indiziertheit reproduktionsmedizinischer Maßnahmen zwischen medical community und rechtlicher, v. a. leistungsrechtlicher Perspektive unterscheidet. Während aus berufsrechtlicher Sicht nichts gegen einen zumindest teilweisen Zugang homosexueller (lesbischer) Paare und unverheirateter Frauen zu ART spricht, ist dieser Zugang leistungsrechtlich verwehrt bzw. uneinheitlich geregelt. Entscheidend ist aus leistungsrechtlicher Sicht nicht die Frage, ob ein Kinderwunsch legitim und somit behandlungsbedürftig ist, sondern wessen Kinderwunsch als legitim und behandlungsbedürftig anzusehen ist. Die hierfür ausschlaggebenden normativen Urteile werden durch den Begriff der Indikation verdeckt, der Objektivität und faktenbasiertes klinisches Urteilen suggeriert. Dadurch entsteht der Widerspruch, dass gerade ART, die zu einer Diversität der Fortpflanzungsmöglichkeiten jenseits des heteronormativen Verständnisses führen können, von traditionellen Ehe- und Familienkonzepten reguliert werden. Hier wirkt eine gesellschaftliche Normierung, die sich in einem Konzept guter Elternschaft manifestiert und sich hinter dem Begriff der Indikation verbirgt.

Danksagung Ich bedanke mich bei Frau Dr. Nadia Primc für hilfreiche Kommentare, die zur Straffung der Argumentation beigetragen haben.

Funding Open Access funding provided by Projekt DEAL.

Open Access Dieser Artikel wird unter der Creative Commons Namensnennung 4.0 International Lizenz veröffentlicht, welche die Nutzung, Vervielfältigung, Bearbeitung, Verbreitung und Wiedergabe in jeglichem Medium und Format erlaubt, sofern Sie den/die ursprünglichen Autor(en) und die Quelle ordnungsgemäß nennen, einen Link zur Creative Commons Lizenz beifügen und angeben, ob Änderungen vorgenommen wurden.

Die in diesem Artikel enthaltenen Bilder und sonstiges Drittmaterial unterliegen ebenfalls der genannten Creative Commons Lizenz, sofern sich aus der Abbildungslegende nichts anderes ergibt. Sofern das betreffende Material nicht unter der genannten Creative Commons Lizenz steht und die betreffende Handlung nicht nach gesetzlichen Vorschriften erlaubt ist, ist für die oben aufgeführten Weiterverwendungen des Materials die Einwilligung des jeweiligen Rechteinhabers einzuholen.

Weitere Details zur Lizenz entnehmen Sie bitte der Lizenzinformation auf http://creativecommons.org/ licenses/by/4.0/deed.de.

\section{Einhaltung ethischer Richtlinien}

Interessenkonflikt G. Rubeis gibt an, dass kein Interessenkonflikt besteht.

Ethische Standards Für diesen Beitrag wurden vom Autor keine Studien an Menschen oder Tieren durchgeführt. Für die aufgeführten Studien gelten die jeweils dort angegebenen ethischen Richtlinien.

\section{Literatur}

Alt-Epping B, Nauck F (2012) Der Wunsch des Patienten - ein eigenständiger normativer Faktor in der klinischen Therapieentscheidung? Ethik Med 24:19-28

Alteri A, Pisaturo V, Nogueira D et al (2019) Elective egg freezing without medical indications. Acta Obstet Gynecol Scand 98:647-652

Beyer DA, Griesinger G, Felberbaum R et al (2013) Fertilitätserhalt in der Onkologie. In: Diedrich K, Ludwig M, Griesinger G (Hrsg) Reproduktionsmedizin. Springer, Berlin, Heidelberg, S 535-544 
Bozzaro C, Krause F, Weisman M (2019) Uterustransplantation. Ethisch gerechtfertigt? Ethik Med. https:// doi.org/10.1007/s00481-019-00519-4

Bundesärztekammer (2018) Richtlinie zur Entnahme und Übertragung von menschlichen Keimzellen im Rahmen der assistierten Reproduktion. Ärzteblatt. https://doi.org/10.3238/arztebl.2018.Rili_ assReproduktion_2018

Buyx A, Hucklenbroich P (2009) „Wunscherfüllende Medizin“ und Krankheitsbegriff: Eine medizintheoretische Analyse. In: Kettner M (Hrsg) Wunscherfüllende Medizin. Ärztliche Behandlung im Dienst von Selbstverwirklichung und Lebensplanung. Campus, Frankfurt/Main, S 25-53

Dörries A (2015) Die medizinische Indikation: Begriffsbestimmung und Rahmenbedingungen. In: Dörries A, Lipp V (Hrsg) Medizinische Indikation. Ärztliche, ethische und rechtliche Perspektiven. Grundlagen und Praxis. Kohlhammer, Stuttgart, S 13-23

Dörries A, Lipp V (Hrsg) (2015) Medizinische Indikation. Ärztliche, ethische und rechtliche Perspektiven. Grundlagen und Praxis. Kohlhammer, Stuttgart

Faircloth C, Gürtin ZB (2018) Fertile connections: thinking across assisted reproductive technologies and parenting culture studies. Sociology 52:983-1000

Gahl K (2005) Indikation - Zur Begründungsstruktur ärztlichen Handelns. Dtsch Med Wochenschr 130:1155-1158

Gemeinsamer Bundesausschuss (G-BA) (2017) Richtlinien des Bundesausschusses der Ärzte und Krankenkassenüber ärztliche Maßnahmen zur künstlichen Befruchtung (,Richtlinien über künstliche Befruchtung“). https://www.g-ba.de/downloads/62-492-1402/KB-RL_2017-03-16_iK-2017-06-02. pdf. Zugegriffen: 28. Mai 2019

Griffiths D (2016) The (re) production of the genetically related body in law, technology and culture: mitochondria replacement therapy. Health Care Anal 24:196-209

Hoffmann B (2017) Biographie und Reproduktionsmedizin. Eine qualitative Studie zum Umgang mit erfolgloser Kinderwunschbehandlung. Pabst Science Publishers, Lengerich

Hunstorfer K, Wallner J (2016) Die Indikation. Medizinethische und rechtsethische Aspekte. Onkologe 22:816-823

Kettner M (2006) „Wunscherfüllende Medizin“ - Assistenz zum besseren Leben? Gesundh Gesell Wiss 6:7-16

Kettner M (2018) Einheit und Differenz wunscherfüllender Medizin. In: Ringkamp D, Wittwer H (Hrsg) Was ist Medizin? Der Begriff der Medizin und seine ethischen Implikationen. Alber, Freiburg, S $19-41$

Krones T, Neuwohner E, El Ansari S et al (2006) Kinderwunsch und Wunschkinder. Möglichkeiten und Grenzen der In-vitro-Fertilisations-Behandlung. Ethik Med 18:51-62

Letherby G (2012) "Infertility" and "involuntary childlessness": Losses, ambivalences and resolutions. In: Earle S, Komaromy C, Layne LL (Hrsg) Understanding reproductive loss: perspectives on life, death and fertility. Ashgate, Farnham, S 9-22

Marckmann G (2015) Wirksamkeit und Nutzen als alternative Konzepte zur medizinischen Indikation. In: Dörries A, Lipp V (Hrsg) Medizinische Indikation. Ärztliche, ethische und rechtliche Perspektiven. Grundlagen und Praxis. Kohlhammer, Stuttgart, S 113-124

Maung HH (2019) Is infertility a disease and does it matter? Bioethics 33:43-53

Maxwell E, Mathews M, Mulay S (2018) More than a biological condition: the heteronormative framing of infertility. Can J Bioeth 1(2):1-98

McTernan E (2015) Should fertility treatment be state funded? J Appl Philos 32:227-240

Möller KH, Makoski K (2020) Rechtliche Regelung der Reproduktionsmedizin in Deutschland. In: Diedrich K, Ludwig M, Griesinger G (Hrsg) Reproduktionsmedizin, 2. Aufl. Springer, Berlin, S 585-614

Montag M, Toth B, Strowitzki T (2020) Polkörper- und Präimplantationsdiagnostik. In: Diedrich K, Ludwig M, Griesinger G (Hrsg) Reproduktionsmedizin, 2. Aufl. Springer, Berlin, Heidelberg, S 269-286

Nationale Akademie der Wissenschaften Leopoldina und Union der deutschen Akademien der Wissenschaften (Hrsg) (2019) Fortpflanzungsmedizin in Deutschland - für eine zeitgemäße Gesetzgebung (2019) Stellungnahme. Nationale Akademie der Wissenschaften Leopoldina und Union der deutschen Akademien der Wissenschaften, Halle

Neitzke G (2015) Medizinische Indikation und ärztliche Indikation. In: Dörries A, Lipp V (Hrsg) Medizinische Indikation. Ärztliche, ethische und rechtliche Perspektiven. Grundlagen und Praxis. Kohlhammer, Stuttgart, S 83-93

van der Ven K, Pohlmann M, Hößle C (Hrsg) (2017) Social Freezing. Die Möglichkeiten der modernen Fortpflanzungsmedizin und die ethische Kontroverse. Springer, Wiesbaden

Wiesing U (2017) Indikation. Theoretische Grundlagen und Konsequenzen für die ärztliche Praxis. Kohlhammer, Stuttgart 
Wilkinson S, Williams NJ (2016) Should uterus transplants be publicly funded? J Med Ethics 42:559-565 Zollner U (2015) Reproduktionsmedizin. In: Kranke P (Hrsg) Die geburtshilfliche Anästhesie. Springer, Berlin, Heidelberg, S 435-450 